\title{
COMPARAÇÃO DE TRÊS MÉTODOS DE REFRIGERAÇÃO DO SÊMEN OVINO PELO PERÍODO DE 24 E 48 HORAS
}

(Comparation of three methods of cooling ovine semen during 24 and 48 hours)

Tacia Gomes Bergstein-Galan, Romildo Romualdo Weiss, Melina A. F. Bertol, Ana Claudia M R Abreu, Amanda Wesolovsky ${ }^{1}$

${ }^{1}$ Correspondência: tacia@alamos.com.br

RESUMO: A manutenção do sêmen ovino refrigerado apresenta vantagens quando comparado ao sêmen fresco e congelado. O objetivo deste trabalho foi avaliar três equipamentos de refrigeração, à $5^{\circ} \mathrm{C}$, do sêmen ovino com base nas análises in vitro da motilidade total, vigor, morfologia espermática e teste hiposmótico (HOST). As amostras seminais foram refrigeradas em geladeira (G1), equipamento automatizado (G2) e dispositivo móvel (G3) durante 24 horas (MI) e 48 horas (MIII). Foi realizado teste de exaustão pela incubação por quatro horas à $37^{\circ} \mathrm{C}$ das amostras mantidas refrigeradas por 24 horas (MII) e 48 horas (MIV). Não houve diferença $(P>0,05)$ entre os equipamentos de refrigeração em todos os parâmetros analisados. Conclui-se que os três métodos de refrigeração foram eficientes em manter os parâmetros in vitro de qualidade espermática similares ao sêmen fresco até 24 horas. Após 48 horas de refrigeração, em todos os métodos, houve decréscimo $(P<0,05)$ da motilidade total, porém sem alteração $(P>0,05)$ do vigor, morfologia espermática ou HOST.

Palavras-chave: carneiro; refrigeração; sêmen

ABSTRACT: The maintenance of chilled sheep semen has advantages when compared to fresh and frozen semen. The objective of this study was to evaluate three cooling equipment, at $5^{\circ} \mathrm{C}$, the ram semen based on in vitro assays of total motility, vigor, morphology and hiposmotic test (HOST). The semen samples were chilled in the refrigerator (G1), automated equipment (G2) and mobile equipment (G3) for 24 hours (MI) and 48 hours (MIII). Depletion test was performed by incubating for four hours at $37^{\circ} \mathrm{C}$ the samples kept refrigerated for 24 hours (MII) and 48 hours (MIV). There was no difference $(P>0.05)$ between the refrigeration equipment in all parameters. We conclude that the three cooling methods were efficient in keeping the parameters of in vitro sperm quality similar to fresh semen up to 24 hours. After 48 hours of cooling in all methods, there was a decrease $(P<0.05)$ of total motility, but no change $(P>0.05)$ for vigor, sperm morphology or HOST.

Key Words: refrigeration; ram; semen 


\section{INTRODUÇÃO}

Após a coleta do sêmen para realização da inseminação artificial, este pode ser utilizado à fresco puro ou diluído, congelado ou resfriado (Maia et al., 2010). O sêmen refrigerado tem como vantagens a facilidade de processamento, armazenamento barato e otimização do uso de reprodutores. Além disso, possibilita a produção de um número maior de doses por ejaculado, sem que haja um comprometimento na concentração final de espermatozoides viáveis por dose inseminante (Vishwanath e Shannon, 2000), além de outras facilidades de logística e armazenamento (Palhão et al., 2006).

Entre as desvantagens do uso do sêmen resfriado, o tempo de uso limitado, horas ou dias, parece ser o principal gargalo Vishwanath; Shannon (2000). O tempo em que o sêmen deverá ser utilizado depende, também, da qualidade espermática inicial, da temperatura de conservação e do diluente empregado (Silva et al., 2002; Severo, 2009). O resfriamento pode provocar redução da motilidade das células, aumento da permeabilidade da membrana plasmática, bem como aumento do número de espermatozoides com movimento circular, lesões no acrossoma e redução da atividade metabólica (Pimenta, 2006).

Várias temperaturas no armazenamento do sêmen refrigerado podem ser utilizadas, entretanto as temperaturas de $15^{\circ} \mathrm{C}$ (Yániz et al., 2005; Druart et al., 2009), $5^{\circ} \mathrm{C}$ (Rojero et al., 2009; Lima et al., 2010) e $4^{\circ} \mathrm{C}$
(Sousa et al., 2010) são as comumente utilizadas. Quando o sêmen é refrigerado, este pode ser armazenado e utilizado até 48 horas após a colheita (Rojero et al., 2009), porém ocorre uma queda acentuada na fertilização após 24 horas na inseminação artificial cervical (Anel et al., 2006; O'Hara et al., 2010).

Dessa forma, o presente estudo visou qualificar através de análises in vitro de motilidade total, vigor, morfologia espermática e teste hiposmótico (HOST), amostras de sêmen ovino refrigerado à $5^{\circ} \mathrm{C}$ em três equipamentos distintos.

\section{MATERIAL E MÉTODOS}

Foram utilizados três carneiros adultos da raça Dorper. A cada semana era realizada a coleta de sêmen dos três animais, com o auxílio de vagina artificial e manequim. Foram realizadas seis coletas em cada reprodutor, com intervalo de uma semana entre elas.

Após cada coleta, as amostras seminais dos três carneiros eram homogeneizadas, formando o pool de sêmen. Os pools eram submetidos à avaliação de coloração, aspecto, volume, motilidade total (MT), vigor, concentração, patologia espermática e HOST, caracterizando o momento 0 (M0). O volume era mensurado, em $\mathrm{mL}$, em copo coletor de vidro graduado.

Após a diluição de $10 \mu \mathrm{L}$ do pool de sêmen em $500 \mu \mathrm{L}$ de Dulbecco modificado Phosphate buffered saline (DMPBS - Nutricell Nutrientes Celulares LTDA, Campinas, Brasil), a análise da motilidade total e vigor era realizada em microscópio óptico com aumento de 400 vezes colocando-se uma gota de sêmen diluído em lâmina sob lamínula, previamente aquecidas a $37^{\circ} \mathrm{C}$. 
Uma alíquota da amostra foi destinada à diluição com DMPBS na concentração de 1:50, com o objetivo de avaliar patologias espermáticas, com 0 método descrito por Cerovsky (1976). A concentração espermática foi realizada em câmara de Neubauer com a razão de diluição de 1:400.

O teste hiposmótico tem como o objetivo de avaliar a integridade da membrana plasmática externa. A solução de trabalho era constituída de água deionizada acrescida de glicose $5 \%$ em partes iguais. Uma alíquota de $10 \mu \mathrm{L}$ do pool de sêmen era diluída em $500 \mu \mathrm{L}$ da solução de trabalho. As amostras eram incubadas por 30 minutos a $37^{\circ} \mathrm{C}$. Após o período de incubação, alíquotas de $10 \mu \mathrm{L}$ das amostras foram colocadas entre lâmina e lamínula, para contagem diferencial de 200 células em microscopia de contraste de fase em objetiva de imersão (Melo e Henry, 1999).

O pool de sêmen foi diluído com o meio glicina-gema-leita (GGL) acrescido de $5 \%$ de gema de ovo (Rodello et al., 2011) até atingir a concentração final de $400 \times 10^{6}$ espermatozóides $/ \mathrm{mL}$. As amostras foram envasadas em palhetas francesas, com 0,25 ml.

As palhetas foram divididas aleatoriamente em três grupos referentes a três métodos distintos de refrigeração: G1 geladeira; G2

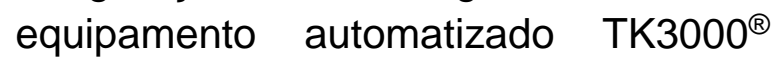
(TK Equipamentos para Reprodução, Uberaba. Minas Gerais, Brasil) e G3 dispositivo móvel de refrigeração (Botutainer $^{\circledR}$ - Botupharma, Botucatu, São Paulo, Brasil.

As amostras referentes ao dispositivo móvel de refrigeração foram colocadas em aparelhos distintos. Isto ocorreu para que não houvesse interferência da possível variação de temperatura nas amostras de 48 horas (MIII) no momento da abertura do dispositivo para a manipulação das amostras em 24 horas (MI).

Para evitar o choque térmico durante o resfriamento na geladeira foi colocada uma bolsa com $400 \mathrm{ml}$ de água a $32^{\circ} \mathrm{C}$ sob as palhetas e duas bolsas com $200 \mathrm{ml}$ cada uma, na mesma temperatura, sobre as amostras. Com 35 e 50 minutos foram retiradas as duas bolsas alocadas sobre as palhetas. Aos 60 minutos foi retirada e de baixo, deixando as palhetas expostas à temperatura da geladeira, segundo método descrito por Rodello (2006).

A MT, vigor, patologia espermática e o HOST foram avaliados após 24 (MI) e 48 (MIII) horas de refrigeração. Foi realizado o teste de exaustão, no qual uma palheta de cada grupo permaneceu em banho-maria a $37^{\circ} \mathrm{C}$ durante quatro horas para análise posterior dos mesmos parâmetros (Paganinni Filho et al., 1997). O momento MII retrata o teste de exaustão das amostras retiradas nas 24 horas e o momento MIV retrata 0 teste de exaustão das amostras retiradas nas 48 horas.

O experimento foi realizado em quintuplicata, com os mesmos animais e mesmos procedimentos. Os dados foram analisados em um delineamento de blocos ao acaso, com três tratamentos (geladeira, equipamento automatizado e dispositivo móvel de refrigeração) e quatro blocos (momentos). Para a análise dos dados foram utilizadas as médias dos valores obtidos nas quintuplicatas de cada tratamento. As variáveis apresentaram distribuição normal e homogeneidade de 
variâncias pelo método KolmorogovSmirnov, sendo utilizado o ANOVA, seguido pelo teste de Tukey, empregando-se 0 programa Action ${ }^{\circledR}$, versão 2.6.216.366 para Windows. Nível de significância de $P>0,05$.

\section{RESULTADOS E DISCUSSÃO}

Os resultados das análises da motilidade total e da patologia espermática estão apresentados na Tabela 1.

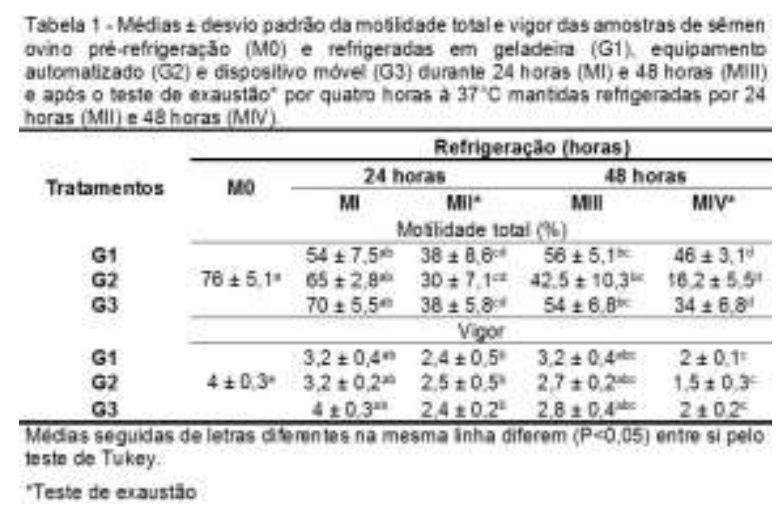

A MT e vigor na amostra inicial obtiveram médias de $76 \pm 5,1^{\text {a }} \%$ e 4 $\pm 0,3^{a}$, respectivamente. A MT não variou $(P>0,05)$ nas amostras refrigeradas à $5^{\circ} \mathrm{C}$ em todos os métodos de refrigeração até as 24 horas, achado similar ao relatado por outros autores (Bucak et al. 2012; Allai et al. 2015). Contudo os dados apresentando na refrigeração à $5^{\circ} \mathrm{C}$ por 24 horas são inconstantes, visto que alguns autores relatam a diminuição da MT ou motilidade progressiva (MP) em outros estudos (Mata-Campuzano et al. 2014; Moscardini et al. (2014), porém estes resultados podem variar dependendo da constituição do meio diluidor Kasimanickam et al. (2011), velocidade de refrigeração (Salamone Maxwell, 2000) ou concentração espermática das amostras Mata-Campuzano et al.
(2015). A MT foi significativamente menor nas 48 horas quando comparadas ao sêmen pré-refrigeração corroborando com outros estudos (Mata-Campuzano et al., 2014; Moscardini et al., 2014; Allai et al., 2015). Segundo Salamon e Maxwell (2000) a MT e integridade espermática diminuem conforme aumenta o tempo de estocagem. Não houve diferença $(P>0,05)$ de MT entre métodos de refrigeração.

A MT foi menor $(P<0,05)$ nos momentos II e IV referentes ao teste de exaustão, esse achado já era esperado e foi observado por outros autores (Falleiros et al., 2013). Os momentos II e IV não diferiram entre si $(P>0,05)$ indicando que o a MT após o período do teste de exaustão não sofreu interferência do período de refrigeração de 24 ou 48 horas.

O vigor não foi influenciado pela refrigeração do sêmen, sendo que não houve diferença significativa nos momentos I e III quando comparado ao momento 0 . Porém houve diferença significativa nos momentos II e IV que foram menores que todos os outros momentos e diferiram entre si $(P<0,05)$. Durante a refrigeração 0 metabolismo celular é reversivelmente inibido (Câmara e Guerra, 2011), como consequência o consumo de substrato presente no meio diluidor diminui. A diminuição do vigor nos momentos referentes ao teste de exaustão pode estar relacionada à disponibilidade de substrato que decresce com o aumento da temperatura de incubação $\left(37^{\circ} \mathrm{C}\right)$. Não houve diferença $(P>0,05)$ entre os grupos estudados.

Kasimanickam et al. (2011) ao refrigerar sêmen ovino à $4^{\circ} \mathrm{C}$ em 
diferentes meios diluidores à base de gema de ovo, leite desnatado e extrato de soja identificou diminuição significativa nos parâmetros cinéticos (VAP, VSL e VCL) após 48 horas de refrigeração, corroborando com os achados do presente trabalho.

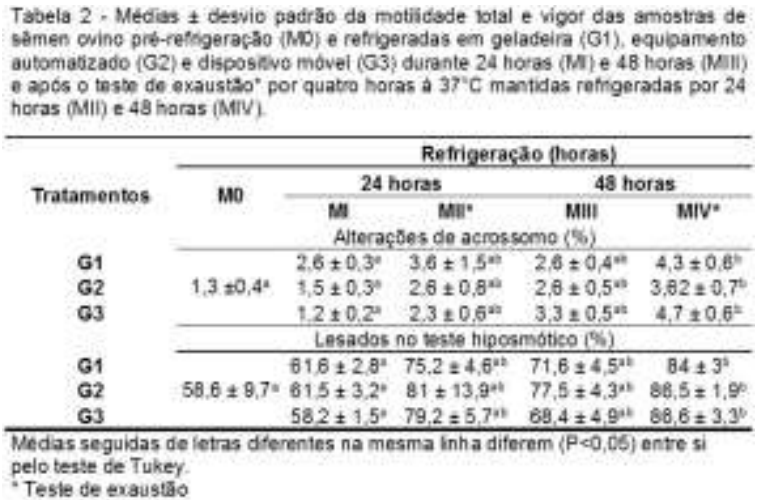

A média inicial da patologia de acrossoma foi de $1,3 \pm 0,4^{\text {a }}$ sendo que não houve diferença significativa nos momentos I, II e III. Porém os momentos 0 e I diferiram do momento IV. Não houve diferença $(P>0,05)$ na patologia de acrossoma entre os métodos de refrigeração. A gema de ovo presente no meio GGL é capaz de proteger as células espermáticas contra o choque térmico, diminui a perda de enzima acrossomicas e previne alterações acrossomática durante a refrigeração espermática (Salamon e Maxwell, 2000).

Outros defeitos espermáticos foram aferidos no experimento, porém não houve diferença $(P>0,05)$ entre os grupos ou momentos estudados.

Com relação ao teste HOST, o valor médio inicial de espermatozoides lesados foi de 58,6 $\pm 9,77^{\mathrm{a}} \%$. O MIV diferiu $(P<0,05)$ dos $M 0$ e $M I$, porém foi similar ao momento MII e MIII.

Quan et al. (2016) ao refrigerar sêmen ovino em meio contendo leite relatou um aumento na concentração de células espermáticas lesadas no teste HOST, diferindo do presente estudo. Porém os resultados não diferiram quando usaram meio diluidor constituído de Tris e Tes.

É possível que no presente estudo as lipoproteínas presentes na gema de ovo foram suficientemente capazes de proteger a membrana plasmática das células e manter sua viabilidade durante o período de 24 horas refrigeração, porém não foi suficiente no período de 48 horas. Esta hipótese é reafirmada pelo aumento $(P<0,05)$ da incidência de células espermáticas com alterações acrossomais no MIV Segundo Salamon e Maxwell (2000) é possível que a estocagem do sêmen refrigerado acelere a maturação das membranas espermáticas aumentando a incidência de espermatozoides com reação acrossomal.

Não houve diferença $(P>0,05)$ entre os métodos de refrigeração em nenhum parâmetro avaliado. Achados similares foram relatados em ovinos (Rodello et al., 2006; Moscardini et al. 2014) e cães (Ferreira et al., 2007) quando comparado diversos equipamentos de refrigeração.

\section{CONCLUSÃO}

Conclui-se que os três métodos de refrigeração foram eficientes em manter os parâmetros in vitro de qualidade espermática similares ao sêmen fresco até 24 horas. Após 48 horas de refrigeração, em todos os métodos, houve decréscimo da motilidade total, porém sem alteração do vigor, morfologia espermática ou HOST. São necessários estudos que verifiquem 


a fertilidade das amostras
acondicionadas em cada um dos
sistemas de refrigeração.

\section{NOTAS INFORMATIVAS}

Os autores declaram que 0 experimento se encontra de acordo com os preceitos da Lei no 11.794, de 8 de Outubro, de 2008, do Decreto no 6.899, de 15 de julho de 2009, e com as normas editadas pelo Conselho Nacional de Controle da Experimentação Animal (CONCEA).

\section{REFERÊNCIAS}

ALLAI, L.; DRUART, X.; CONTELL, J.; et al. Effect of argan oil on liquid storage of ram semen in Tris or skim milk based extenders. Animal reproduction science, v. 160, p. 57-67, 2015.

ANEL, L.; ALVAREZ, M.; MARTINEZPASTOR, F.; GARCIA-MACIAS, V.; et al. Improvement strategies in ovine artificial insemination. Reproduction in Domestic Animals, v. 41, supl. 2, p. 3042, 2006.

BUCAK, M. N.; COYAN, K.; OZTÜRK, C.; GÜNGÖR, S.; OMÜR, A. D. Methionine supplementation improves ram sperm parameters during liquid storage at $5^{\circ} \mathrm{C}$. Cryobiology, v. 65, n. 3, p. 335-7, 2012.

CÂMARA, D. R.; GUERRA, M. M. P. Refrigeração e criopreservação do sêmen ovino: danos inerentes à técnica e influência da suplementação do meio com antioxidantes sobre a qualidade espermática. Revista Brasileira de Reprodução Animal, p. 33-40, 2011.

CEROVSKY, J.A. A new staining procedure for boar spermatozoa. Zivocisna Vyroba, v. 21, p. 351-362, 1976.

DRUART, X.; COGNIÉ, J.; BARIL, G.; CLÉMENT, F.; et al. In vivo imaging of in situ motility of fresh and liquid stored ram spermatozoa in the ewe genital tract. Reproduction, v. 138 , p. 45-53, 2009.

FALLEIROS, M. B.; BICUDO, S. D.; RODELLO, L.; et al. Implicações do uso do extrato de LBD (Lipoproteínas de baixa densidade) ou da gema de ovo "purificada", sobre a motilidade e morfologia espemática no sêmen ovino refrigerado por 24 ou 48 horas. Veterinária e Zootecnia, v. 20, n. (2), p. 307-317, 2013.

FERREIRA, M. A.; BARCELOS, B. P.; JAMAS, L.; et al. Efeito da unidade de resfriamento e conservação, do tempo de armazenamento e diluidor sobre as características do sêmen de cães Effect of the chilling sistemas, time of conservation and extender on dog.' $s$ semen characteristics. Revista Brasileira de Ciencias Veterinárias, v. 14, n. 2, p. 86-90, 2007.

KASIMANICKAM, R.; KASIMANICKAM, V.; TIBARY, A.; PELZER, K. Effect of semen extenders on sperm parameters of ram semen during liquid storage at $4^{\circ} \mathrm{C}$. Small Ruminant Research, v. 99, n. 2-3, p. 208-213, 2011.

LIMA, L. F.; MOURA, P.; PASSOS, P. I. B.; LEAL, D. R.; et al. Influência de sistemas de refrigeração sobre a qualidade do sêmen ovino criopreservado em palhetas. Ciência Animal Brasileira, v. 11, n. 4, p. 835844, 2010.

MAIA, M. D. S.; BICUDO, S. D.; SICHERLE, C. C.; RODELLO, L.; GALLEGO, I. C. S. Lipid peroxidation and generation of hydrogen peroxide in frozen-thawed ram semen cryopreserved in extenders with antioxidants. Animal Reproduction Science, v. 122, n. 1-2, p. 118-123, 2010.

MATA-CAMPUZANO, M.; ÁLVAREZRODRÍGUEZ, M.; TAMAYO-CANUL, J.; et al. Refrigerated storage of ram sperm in presence of Trolox and $\mathrm{GSH}$ 
antioxidants: Effect of temperature, extender and storage time. Animal Reproduction Science, v. 151, n. 3-4, p. 137-147, 2014.

MATA-CAMPUZANO,

M.;

SOLEILHAVOUP, C.; TSIKIS, G.; et al. Motility of liquid stored ram spermatozoa is altered by dilution rate independent of seminal plasma concentration. Animal reproduction science, v. 162, p. 31-6, 2015.

MELO, M. I. V.; HENRY, M.; BEKER, A. R. C. L. Teste hiposmótico para avaliação da viabilidade do sêmen eqüino resfriado com diferentes diluidores. Arquivo Brasileiro de Medicina Veterinária e Zootecnia, v. 57, p. 757-763, 2005.

MOSCARDINI, M. M.; SCOTT, C.; MOURA, D. S.; et al. Viabilidad de espermatozoides ovinos mantenidos a 50 y $150 C$ en diferentes sistemas de refrigeración. Revista Brasileira de Ciência Veterinárias, v.21, p. 122-126, 2014.

O'HARA, L.; HANRAHAN, J. P.; RICHARDSON, L.; DONOVAN, A.; et al. Effects of storage duration, storage temperature, and diluent on the viability and fertility of fresh ram sperm. Theriogenology, v. 73, p. 541-549, 2010.

PAGANINI, FILHO, P.; BICUDO, S.D.; SOUZA, M.I.L.; SOUSA, D.B. Viabilidade do sêmen ovino frente a três diluidores em temperaturas de $37^{\circ} \mathrm{C}$ e sob refrigeração. In: Congresso Brasileiro de Reprodução Animal, XII, Belo Horizonte, 1997.

PALHÃO, M. P.; BISPO, C. A. S.; FURST, R. ; ROVAY, H.; CARVALHO, G. R.; BISPO, M. S. Efeito de diferentes curvas de resfriamento e diluentes na conservação do sêmen caprino. Acta Scientiae Veterinariae, v. 34, p. 571571, 2006.

PIMENTA, S.A. Inseminação artificial em caprinos com sêmen resfriado. 2006. Belo Horizonte, 48f. Dissertação de Mestrado, Universidade Federal de Minas Gerais Escola de Veterinária.

QUAN, G. B.; WU, G. Q.; WANG, Y. J.; et al. Effects of the Tris, Tes, or skim milk based extender on in vitro parameters of ram spermatozoa during liquid storage. Small Ruminant Research, v. 134, p. 14-21, 2016.

RODELLO, L. Validação de sistema automatizado de refrigeração e congelação de sêmen ovino. 2006. Botucatu, 86f. Dissertação (Mestrado em Medicina Veterinária) - Curso de Pós Graduação em Medicina Veterinária, Faculdade de Medicina Veterinária e Zootecnia, UNESP.

RODELLO, L.; BICUDO S.D.;FALLEIROS, B.M;MONTEIRO, D.C.; SAKASHITA, M.S. Implicações da redução na concentração de gema de ovo no meio glicina-gema-leite sobre a cinética, morfologia e integridade de membranas espermáticas em sêmen ovino criopreservado. Veterinária e Zootecnia, v. 18(2), p. 239-248, 2013

ROJERO, R. D. M.; REYNASANTAMARIA, L.; MICHEL-ACEVES, A. C.; MASTACHE-LAGUNAS, A. A.; et al. Cervical or intrauterine artificial insemination in pelibuey ewes, with chilled semen. Journal of Animal and Veterinary Advances, v. $8, n$. 12, p. 2621-2625, 2009.

SALAMON, S.; MAXWELL, W. M. C. Storage of ram semen. Animal Reproduction Science, v. 62, n. 1-3, p. 77-111, 2000.

SEVERO, C. K. Avaliação da adição de cisteína no sêmen resfriado para a inseminação em suínos. 2009. Santa Maria, 93f. Dissertação (Mestrado em Medicina Veterinária), Universidade Federal de Santa Maria - Rio Grande do Sul.

SILVA, L. D. M. da.; SILVA. A. R.; CARDOSO, R. de C. S. Em: GONSALVES, P. B. D.; FIGUEREDO. J. R. de.; FREITAS, V. J. de. F. (Eds). Biotécnicas aplicadas à reprodução animal. São Paulo: Varela, 2002. 
VISHWANATH, R.; SHANNON, P. Storage of bovine semen in liquid and frozen state. Animal Reproduction Science, v. 62, n. 1-3, p. 23-53, 2000. 13

YÁNIZ, J.; MARTÍ, J. I.; SILVESTRE, M. A.; FOLCH, J.; et al.Effects of solid storage of sheep spermatozoa at $15^{\circ} \mathrm{C}$ on their survival and penetrating capacity. Theriogenology, v. 64, p. 1844-1851, 2005. 